\title{
Fortalecimiento de la Red de Mujeres Rurales mediante la producción, procesamiento y comercialización de granos básicos
}

\author{
Strengthening the Network of Rural Women through \\ the production, processing and marketing of basic grains
}

\author{
Alejandra Bonilla Leiva' \\ Ana Ruth Bonilla Leiva² \\ Marvin Amador Guzmán ${ }^{3}$ \\ Nancy Hidalgo Dittel ${ }^{4}$
}

Fecha de recepción: 20 de enero del 2012

Fecha de aprobación: 19 de marzo del 2012

Este artículo cuenta con el aval de la

Vicerrectoría de Investigación y Extensión del

Tecnológico de Costa Rica
Licenciada en Administración de Empresas Agropecuarias. Escuela de Ciencias Agrarias, Facultad de Ciencias de la Tierra y el Mar, Universidad Nacional. Teléfono: 2277-3504. Correo electrónico: bonilla.alejandra@gmail.com. Ciencia y Tecnología de Alimentos, Universidad de Costa Rica. Teléfono: 25 I I-3592. Correo electrónico: ana.bonillaleiva@ucr.ac.cr. Máster en Comunicación. Escuela de Ciencias de la Comunicación Colectiva, Universidad de Costa Rica. Teléfono: 8335-3070. Correo electrónico: marvin.amador@gmail.com

4 Máster en Manejo de Recursos Naturales. Escuela de Ingeniería Agrícola, Tecnológico de Costa Rica. Teléfono 2550-227I. Correo electrónico: nhidalgo@itcr.ac.cr 


\section{Resumen}

El presente proyecto ha logrado incrementar la producción de granos básicos, su industrialización y comercialización para tener alternativas alimentarias y nutritivas, de modo que se fortalezca la soberanía alimentaria de las zonas rurales de Costa Rica.

El impacto en lo local se expresa en el fortalecimiento de los ingresos familiares de las personas involucradas en el proyecto, el incremento de la producción de granos, la industrialización producto de la transferencia de tecnología, el mejoramiento en la dieta y la generación de empleo para las mujeres, tanto en la producción de granos en el campo como en el manejo poscosecha y el procesamiento para la elaboración de productos empacados para el mercado.

La generación de capacidades en las asociadas a la Red de Mujeres Rurales y el fortalecimiento de esta son logros del compromiso de las mujeres y el apoyo constante del proyecto.

Por otra parte, se generó un producto a base de frijol y arroz con un alto valor nutritivo, que se ha vendido en ferias y algunos pequeños locales comerciales, y que se podrá distribuir en escuelas, centros infantiles y abastecedores locales. El impacto regional se da en la introducción del producto, cuyo principal consumidor meta es la población infantil y juvenil, ya que se busca mejorar sus hábitos alimenticios; posteriormente, se pretende extender al mercado nacional.

\section{Palabras clave}

Mujeres, granos básicos, semillas, procesamiento de granos, comercialización, desarrollo rural, seguridad alimentaria.

\begin{abstract}
This project was able to increase grain production, industrialization and marketing, to have food and nutrition alternatives to strengthen the food security of rural areas. The local impact is expressed in strengthening family income of the people involved in the project, the increase in grain production, industrialization technology transfer, diet improvement and employment generation for women, both in grain production in the field and in food processing.

Capacity building in women associated to Rural Women's Network was possible because of women's commitmentand the continued support of the project. A product based on beans and rice with a high nutritional value was generated, which could be distributed in schools, childcare centers and local suppliers. The regional impact is given in the product introduction with emphasis on children. Then, it will be extended to larger market.
\end{abstract}

\section{Key words}

Women, grains, seeds, grain processing, marketing, rural development, food security. 


\section{Marco teórico}

\section{Seguridad alimentaria}

La Declaración Universal de los Derechos Humanos de 1948 (Naciones Unidas, 1948), en el artículo 25, establece que:

Toda persona tiene derecho a un nivel de vida adecuado que le asegure, así como a su familia, la salud y el bienestar, y en especial la alimentación, el vestido, la vivienda, la asistencia médica y los servicios sociales necesarios; tiene asimismo derecho a los seguros en caso de desempleo, enfermedad, invalidez, viudez, vejez y otros casos de pérdida de sus medios de subsistencia por circunstancias independientes de su voluntad.

La situación de inseguridad alimentaria y nutricional se tornó más compleja a partir del año 2002, debido al aumento en los precios de los alimentos en los mercados locales e internacionales. Por otra parte, el impacto de la crisis financiera y económica que desacelera el crecimiento mundial, ha incrementado el desempleo y la pobreza. Desde 1993, en la región centroamericana los jefes de Estado y ministros de Salud,Agricultura y Ambiente establecieron una serie de compromisos en forma de acuerdos, agendas y planes relacionados con el fortalecimiento de la seguridad alimentaria y nutricional. Un documento básico es el Plan de Centroamérica y República Dominicana 20I2-20I5, que posiciona el tema de seguridad alimentaria y nutricional como un eje transversal para el cumplimiento de los objetivos del milenio (ODM) y para el desarrollo social y económico de los países de la región (Ministerio de Salud, 20 I I).

Centroamérica es una región con una elevada proporción de población rural, en comparación con el resto de América Latina: la población rural centroamericana representa el 4 I,4 I\% de la población total, mientras que la media latinoamericana se sitúa en el $20,48 \%$ del total.

La subnutrición se refiere a la insuficiencia permanente de alimentos en cantidad y calidad adecuadas para satisfacer las necesidades energéticas de la población. Centroamérica presenta un porcentaje de personas subnutridas (I4,2\%), seis puntos porcentuales por encima de la media de América Latina y el Caribe (9\%), por lo que constituye una de las zonas más vulnerables en cuanto a subnutrición dentro del continente americano. Centroamérica se encuentra en un estado nutricional de transición, donde coexisten las deficiencias nutricionales y la obesidad, debido a una mala calidad de la dieta.

\section{Productores de granos básicos}

La agricultura familiar, en sentido amplio, está compuesta en Centroamérica por al menos 2350000 familias. El peso económico representa alrededor del $50 \%$ del sector agropecuario. Los principales actores de la agricultura familiar en Centroamérica son los productores y productoras de granos básicos. Los productores de granos básicos en Centroamérica superaron los 2 millones (2 064 000) entre el 2005 y el 2007, e involucran directamente, a través de sus familias, a casi 12 millones de personas, de las cuales más de 10 millones serían rurales. La disponibilidad media de tierra de los hogares para el cultivo de granos básicos pasó de I,8 hectáreas en 1987, a I,4 hectáreas en 2007, es decir, hubo una reducción del $22 \%$.

Las características de los hogares productores de granos básicos en Centroamérica son las siguientes (FAO-RUTA, 20 I0):

- 6 de cada 10 hogares padecen inseguridad alimentaria nutricional.

- Más del 50\% de los hogares productores de granos básicos tiene algún miembro trabajando como asalariado permanente.

- La reducción en la producción de granos básicos fue de 19,8 kilos por habitante entre 1970 y 2007.

- Hay cerca de 12 millones de personas agrupadas en familias con una media de 5,4 miembros.

- El 85\% de los hogares tiene una jefatura masculina y un promedio de 49 años de edad.

- En El Salvador, Guatemala, Honduras y Nicaragua se cuenta con 2 años promedio de educación formal recibida, mientras que en Costa Rica y Panamá son 3 años.

- El 63\% de las explotaciones son de tenencia propia, mientras que el $37 \%$ son ajenas.

- Se cuenta con un 34\% en pobreza y $32 \%$ en pobreza extrema (PRESANCA y FAO, 20l I). 


\section{Política costarricense relacionada}

En Costa Rica se han elaborado diversas políticas y planes para abordar el tema de la seguridad alimentaria. El Plan Nacional de Salud 201 0-202 I para Costa Rica establece como objetivo la promoción de la seguridad alimentaria y nutricional mediante sistemas de producción, comercialización y consumo de alimentos propiciadores de la salud, bajo criterios de inocuidad y sostenibilidad socio-ambiental, así como la mejora del estado nutricional de la población en general, con énfasis en grupos en condición de pobreza, vulnerabilidad y exclusión. Otro objetivo de dicho plan es la promoción de la infraestructura física para la producción, conservación, distribución, preparación y consumo de alimentos con el fin de garantizar una seguridad alimentaria y nutricional como derecho de la población (Ministerio de Salud, 2010).

La Política Nacional para la Seguridad Alimentaria y Nutricional 20 I I-202I (Ministerio de Salud, 20 I I) establece una serie de asuntos críticos, políticas

\begin{tabular}{|c|c|c|}
\hline \multicolumn{3}{|c|}{$\begin{array}{l}\text { Ámbito: Disponibilidad de alimentos } \\
\text { Área de intervención: producción alimentaria }\end{array}$} \\
\hline Asuntos críticos & Políticas & Estrategia \\
\hline $\begin{array}{l}\text { Crisis alimentaria, falta } \\
\text { de crédito oportuno, } \\
\text { crisis energética, crisis } \\
\text { económica, cambio } \\
\text { climático. }\end{array}$ & $\begin{array}{l}\text { Fomento de la } \\
\text { producción de alimentos } \\
\text { con calidad e inocuidad } \\
\text { para la población. } \\
\text { Adaptación de los } \\
\text { procesos productivos al } \\
\text { cambio climático. }\end{array}$ & $\begin{array}{l}\text { Producción de alimentos con calidad e inocuidades básicas y nutritivas, de } \\
\text { acuerdo con la demanda interna y las condiciones climáticas (zonificación). } \\
\text { Programa de mitigación y recuperación agropecuaria para atender los } \\
\text { efectos de las emergencias y desastres producidos por el cambio climático. } \\
\text { Investigación en nuevas tecnologías que mejoren la producción de } \\
\text { alimentos, con calidad e inocuidad,y sin detrimento de los recursos } \\
\text { naturales. }\end{array}$ \\
\hline $\begin{array}{l}\text { Producción limitada de } \\
\text { granos básicos, frutas y } \\
\text { vegetales. }\end{array}$ & $\begin{array}{l}\text { Fomento de la } \\
\text { producción nacional } \\
\text { para la disponibilidad de } \\
\text { alimentos con énfasis en } \\
\text { granos básicos, vegetales } \\
\text { y frutas de manera } \\
\text { sostenible, segura y } \\
\text { competitiva. }\end{array}$ & Incremento de la productividad y la competitividad de la agricultura familiar. \\
\hline \multicolumn{3}{|c|}{$\begin{array}{l}\text { Ámbito: Disponibilidad de alimentos } \\
\text { Área de intervención: comercialización }\end{array}$} \\
\hline Asuntos críticos & Políticas & Estrategia \\
\hline $\begin{array}{l}\text { Los pequeños y medianos } \\
\text { productores no cuentan } \\
\text { con una infraestructura } \\
\text { básica (equipamiento, } \\
\text { almacenamiento, caminos } \\
\text { de acceso, transporte). }\end{array}$ & $\begin{array}{l}\text { Abogacía para el } \\
\text { mejoramiento de las } \\
\text { condiciones básicas } \\
\text { para la comercialización } \\
\text { eficiente y eficaz de la } \\
\text { producción de alimentos. }\end{array}$ & $\begin{array}{l}\text { Habilitación de la infraestructura rural, con énfasis en los cantones } \\
\text { prioritarios. } \\
\text { Desarrollo de programas de acopio y de almacenamiento de la } \\
\text { producción. } \\
\text { Programa de manejo adecuado de los productos poscosecha. } \\
\text { Fortalecimiento de la capacidad empresarial y de gestión de la producción } \\
\text { de productores organizados, con énfasis en los cantones prioritarios. }\end{array}$ \\
\hline $\begin{array}{l}\text { Falta de estrategias } \\
\text { de mercadeo para la } \\
\text { comercialización de la } \\
\text { producción nacional. }\end{array}$ & $\begin{array}{l}\text { Fomento de estrategias } \\
\text { de mercadeo para la } \\
\text { comercialización de la } \\
\text { producción nacional. } \\
\text { Promoción de sistemas } \\
\text { de comercialización } \\
\text { alternativos de alimentos. }\end{array}$ & $\begin{array}{l}\text { Alianzas entre sectores para la integración y el acceso a los mercados. } \\
\text { Integración y formación de cadenas agro productoras de alimentos con } \\
\text { calidad e inocuidad en todas sus etapas para asegurar la producción de } \\
\text { alimentos para consumo interno y de exportación. } \\
\text { Creación de condiciones para que las organizaciones de productores } \\
\text { dispongan de capacidades para la comercialización de productos } \\
\text { (CEPROMAS, modernización de las ferias del agricultor). } \\
\text { Promoción de la existencia de reservas alimentarias nacionales y locales. } \\
\text { Fomento de la agroindustria a nivel local para generar mayor valor } \\
\text { agregado. } \\
\text { Fomento a la comercialización de la producción nacional a través de sellos } \\
\text { verdes, denominación de origen,certificados de calidad e inocuidad, y } \\
\text { nuevos canales de comercialización. }\end{array}$ \\
\hline
\end{tabular}




\begin{tabular}{|l|l|l|}
\hline \multicolumn{3}{|l|}{ Ámbito: Consumo de alimentos } \\
Área de intervención: hábitos alimentarios y estilos de vida saludables \\
\hline Asuntos críticos & Políticas & Estrategia \\
\hline $\begin{array}{l}\text { Hábitos alimentarios y } \\
\text { estilos de vida afectan } \\
\text { negativamente la salud y } \\
\text { nutrición de la población. }\end{array}$ & $\begin{array}{l}\text { Promoción de hábitos y } \\
\text { estilos de vida saludables } \\
\text { que permitan mejorar } \\
\text { la salud y nutrición } \\
\text { de la población, para } \\
\text { lo que se tomarán en } \\
\text { cuenta lineamientos } \\
\text { recomendados a nivel } \\
\text { nacional e internacional. }\end{array}$ & $\begin{array}{l}\text { Impulios de la parida saludable. } \\
\text { estilos los actores sociales en la promoción de } \\
\text { Fomento del consumo de alimentos tradicionales por región. }\end{array}$ \\
\hline
\end{tabular}

y estrategias relacionadas con el tema de este proyecto, tales como:

El Plan Nacional de Alimentos que ha planteado el Gobierno ante la crisis mundial alimentaria, señala que las respuestas a la problemática internacional no pueden ser solo paliativas y a corto plazo. El país debe prepararse adecuadamente para establecer las bases que garanticen la seguridad alimentaria y nutricional de la población a largo plazo, y reducir su vulnerabilidad.

La producción de alimentos básicos, en muchas de las áreas anteriormente productoras de granos, ha sido sustituida por productos de exportación o de consumo interno, menos riesgosos, más rentables y competitivos. Un grupo importante de productores se ha reubicado en este tipo de agricultura, mientras que otro grupo se ha trasladado a otros sectores de la economía, como el turismo, la industria, el comercio y los servicios.

Es por ello que un plan de reactivación de la producción de alimentos, entre ellos los granos, debe considerar, en primer lugar, la disponibilidad de áreas para la siembra, así como productores dispuestos a incursionar de nuevo en la producción de estos granos, o productores que se han mantenido en la actividad y que estén dispuestos a aumentar sus áreas.

El plan tiene como objetivo general que la población, en especial los grupos vulnerables, disponga, acceda y consuma alimentos inocuos y nutritivos, mediante el compromiso y la articulación de la institucionalidad pública, sector privado, organizaciones de productores y otros actores de la sociedad. Para alcanzar el objetivo general, se operará con tres componentes: disponibilidad, acceso a los alimentos y combate de la pobreza, los cuales se configuran en los siguientes objetivos específicos:
1. Asegurar la disponibilidad de alimentos básicos (maíz, arroz, frijoles y otros productos).

2. Garantizar el acceso de alimentos a los grupos vulnerables, con el fin de mejorar su seguridad alimentaria y nutricional.

3. Evitar el incremento de los niveles de pobreza ante los aumentos de los precios de los productos de la canasta básica alimentaria (SEPSA, 2008).

A pesar de las políticas y planes propuestos y relacionados con la seguridad alimentaria, en diversas regiones del país se continúa extendiendo la producción a gran escala de productos para la exportación, especialmente banano, piña y algunos tubérculos. En forma paralela, se han aprobado políticas dirigidas a promover la producción para el mercado externo, lo que provoca que las dinámicas socio-productivas generadas se caractericen por una fuerte tendencia a la concentración de la tierra en manos de grandes propietarios, la mayoría de origen transnacional, así como por una proletarización del pequeño productor. Todo esto ha implicado que la producción de granos básicos, otrora con un peso significativo en el balance productivo, haya quedado marginada a pesar de que constituya la base de la alimentación de la mayoría de la población del país y, particularmente, de las poblaciones rurales.

\section{Aumento de la pobreza en Costa Rica}

El XVII Estado de La Nación (Conare, 20I I) señala que la pobreza afectó a un 21,3\% de los hogares en el 2010 . Tanto este resultado como los indicadores que dan cuenta de la pobreza extrema, la intensidad (o brecha) y la severidad de la pobreza superan los niveles reportados en las últimas encuestas de hogares. Además, un 13,4\% de los hogares no pobres se encuentra en condición de vulnerabilidad ante la pobreza, de modo que, al sumar esta cifra a la 
de hogares pobres, se obtiene un 35\% de las familias costarricenses. Las brechas entre zonas y entre regiones se mantuvieron elevadas, en detrimento de la zona rural y las regiones periféricas, en especial la brunca y la chorotega.

En términos absolutos, mientras en el 2009 había 236800 hogares pobres, en el 2010 el número aumentó a 274616 hogares, de los cuales 77365 vivían en pobreza extrema. Esto representa un aproximado de I 103522 personas en pobreza total y 31 I 03 I en pobreza extrema $(24,2 \%$ y $6,8 \%$ de la población total). Estas cifras son las más altas de la década. Además, la diferencia en la pobreza total por zona pasó de 4 a 8 puntos porcentuales entre 2009 y 20 I 0. La ampliación de la brecha es producto de una disminución en la incidencia de la pobreza urbana (de 19\% a 18,3\%) y un aumento en la rural (de 23\% a 26,3\%).

Tales resultados indican que el sector rural es el que más sufre las consecuencias del frágil desempeño macroeconómico del país, caracterizado por un bajo o moderado crecimiento, la débil generación de empleo y una reducida expansión en los ingresos reales. En materia de desigualdad de ingresos, las últimas ediciones de este informe pusieron en evidencia que Costa Rica pasó de una situación que la acercaba a las naciones desarrolladas, a una más semejante a la de los países latinoamericanos, pues, en la primera década del siglo XXI, la desigualdad medida por el coeficiente de Gini mostró niveles claramente ascendentes y superiores a los observados en la década previa.

Los cálculos realizados a partir de la encuesta nacional de hogares 2010 no solo confirman las tendencias reportadas, sino que además revelan que la concentración del ingreso prevaleciente en el país es mucho mayor de lo que se creía.

Otro tema de interés es el acceso desigual a las oportunidades por razones de género. Luego de cuatro años de la puesta en ejecución de la Política Nacional para la Igualdad y Equidad de Género (PIEG), la evolución de los indicadores de seguimiento para el periodo 2007-2010 revela que, si bien se registraron progresos en algunos ámbitos, estos fueron selectivos y lentos. Además, se constata un desarrollo muy desigual entre objetivos, pues los logros y los desafíos pendientes muestran una alta variabilidad. Los mayores rezagos se observan en materia de empleo, cuido y violencia contra las mujeres.

Las mujeres siguen mostrando tasas de participación y ocupación menores que las de los hombres; su tasa de desempleo en el 2010 fue 3,5 puntos porcentuales superior a la de los hombres.

Asimismo, se señala en dicho Informe que, en términos comparativos, el ritmo de recuperación de la economía costarricense fue menor que el mundial (5\%) y que el del grupo de países en desarrollo y emergentes ( $3 \%$ y $7,3 \%$, respectivamente); incluso fue inferior a la expansión observada en América Latina y el Caribe, donde el crecimiento alcanzó un 6,1\% en el 2010 , sobre todo por el dinamismo que mostraron Argentina (9,2\%), Brasil (7,5\%), Perú $(8,8 \%)$ y Uruguay (8,5\%), cuyas tasas fueron superiores al $7 \%$.

\section{Industrialización de granos básicos}

La Universidad de Costa Rica, por medio del CITA, durante el periodo 2002-2007, como parte del Programa Colaborativo de Apoyo a la Investigación Frijol/Frijol de Ojo Negro (CRSP-USAID-donación $N^{\circ}$, DAN-G-SS-86-0008-88), desarrolló ingredientes y productos finales a partir de frijol, con características de alimentos funcionales, con un gran potencial de mercado (Bonilla y Nielsen, 2007).

Entre los productos desarrollados se encuentran las galletas de frijol. Este producto fue evaluado a través de un análisis de aceptación de consumidores y quedó demostrada su alta aceptación. Por otra parte, las galletas fueron evaluadas químicamente para determinar los parámetros nutricionales. Los resultados mostraron que las galletas contienen 5\% proteína, 8\% lípidos, 3,4\% fibra y 2,44\% almidón resistente, $y$ son una fuente de actividad antioxidante 36,7 mg Trolox/g. También se estimó que, con base en la textura de las galletas, la vida útil a temperatura ambiente es de al menos 160 días, utilizando polipropileno biorientado como empaque (Soto, 2008).

Adicionalmente a los estudios realizados, la Universidad de Costa Rica, por medio de PROINNOVA, diseñó y registró la marca "Pinticas", la cual fue licenciada a los grupos de mujeres que elaboran las galletas (figura I). 


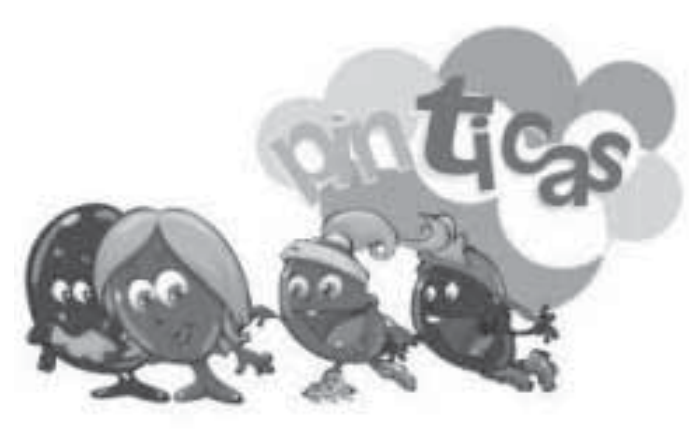

Figura I. Marca registrada para las galletas de frijol y arroz.

\section{Enfoque del proyecto}

El proyecto retoma dos líneas de trabajo fundamentales en el desarrollo rural: el fortalecimiento de la producción de alimentos y el fortalecimiento de las mujeres como actoras de los procesos económicos, al igual que su participación en la construcción del nuevo escenario rural, tradicionalmente invisibilizadas en las dinámicas económicas. La nueva ruralidad nos obliga a la interrelación de factores productivos, sociales, ambientales y culturales.

La crisis mundial de alimentos reposiciona la necesidad de la producción local de alimentos en las comunidades rurales, las cuales han sido excluidas de la bonanza económica de la transnacionales. Ante las alzas en los precios internacionales de los productos básicos, cereales y leguminosas, las poblaciones de menores recursos ya empiezan a sentir el impacto en la reducción del consumo. Esta situación nos plantea el desafío del incremento de la producción de alimentos en manos de las familias campesinas, a fin de mitigar el impacto de la escasez derivada de los precios altos; pero se articula la producción con la conservación de las reservas genéticas y el procesamiento.

Actualmente, en el desarrollo de las comunidades rurales y las economías campesinas no es suficiente el estímulo de la producción, se necesita el procesamiento o la agroindustrialización a fin de lograr productos de mayor calidad, de más fácil consumo y que aporten mayores ingresos. Por otra parte, en la preparación de los alimentos, las mujeres han participado históricamente sin reconocimiento, por lo que la agroindustrialización permite valorar económica y socialmente esa contribución de las mujeres, "...la actividad agroindustrial permite demandar mano de obra adicional para las diversas etapas del proceso productivo, representando una posibilidad de trabajo para otros miembros de la familia, donde las mujeres juegan un rol fundamental" (Rebosio y Melgar, 200 I).

Esto nos ubica en la perspectiva de equidad de género en el Desarrollo Rural: "En el análisis de las diferencias de género, estamos en un continuo ir y venir en relación con la posesión de la propiedad, los recursos, las posibilidades de producir, del control de capital, en fin del "patrimonio productivo" (Bonilla, 2007).

Por ello, partimos del siguiente principio: El acceso a los recursos económicos, ya sea vía salarios o posesión del ciclo productivo, no conduce per se a la emancipación de las mujeres, pero no hay emancipación sin control de los recursos, por ello le concedemos una importancia de primer orden a la posesión y el control del patrimonio productivo (Bonilla, 2007).

La falta de opciones laborales, obliga al campesinado a convertirse en empleado de las compañías que ahora concentran las tierras, otrora de las familias campesinas. Esta dinámica de pérdida de la tierra no deja espacio para que las comunidades determinen sus perspectivas de desarrollo, elimina la posibilidad de decidir sobre la producción, no solo se traduce en la pérdida de sus tierras, sino en los recursos patrimoniales que le acompañan, como vivienda, fuentes de agua, biodiversidad, interrelaciones con la naturaleza y en muchos casos aire limpio (Bonilla, 2007).

La racionalidad campesina, que contempla muchos más aspectos que la dinámica económica del trabajo por salario, ha forjado una relación con el entorno que, en términos amplios, significan recursos económicos aunque no necesariamente monetarios, por consiguiente, ha conformado una cultura que se dimensiona desde la satisfacción de las necesidades alimentarias hasta el esparcimiento. Las mujeres, en su rol, participan en estas dinámicas de manera muy particular, pero, al ver reducido su patrimonio, viven el deterioro de sus condiciones de vida y de sus relaciones con el entorno social y ambiental. El siguiente esquema plantea el árbol de problemas detectados. 


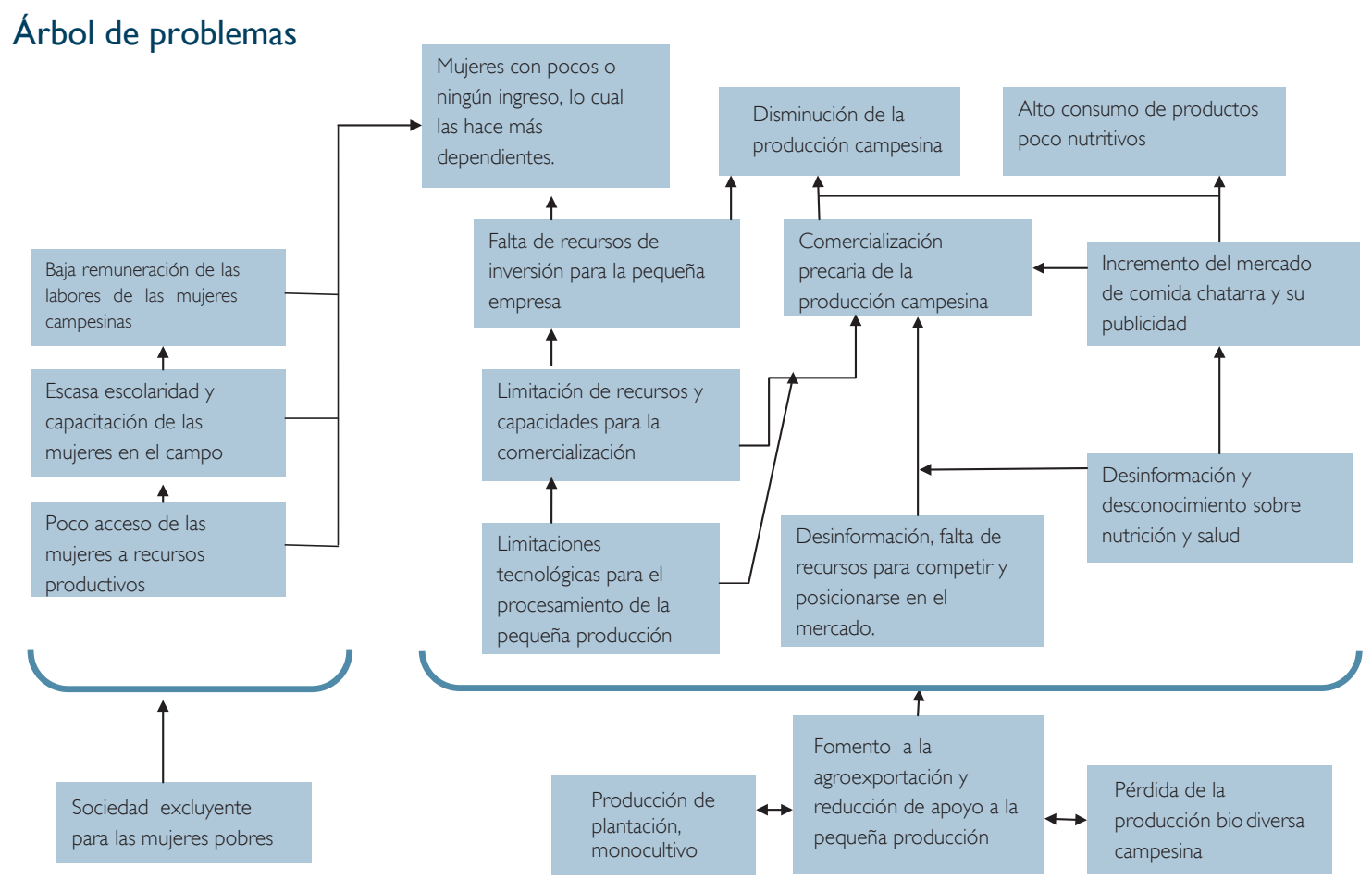

Fuente: Bonilla, 2007

\section{Red de Mujeres Rurales}

La Red de Mujeres Rurales, organización de base que cuenta con grupos en todo el país, se formó en agosto del 2006 y desde entonces ha fortalecido los procesos de organización de las mujeres rurales para buscar opciones de producción, generación de ingresos y defensa de sus intereses y derechos. Durante los últimos dos años, algunos grupos en la Red de Mujeres Rurales han fortalecido o retomado la producción de granos básicos, mientras que otros han dado pasos en la búsqueda de recursos para establecer el procesamiento artesanal de alimentos. En este proyecto se trabajó con los grupos de Los Chiles, Cariari y El Águila de Pérez Zeledón.

\section{Metodología}

Fortalecimiento de la producción de granos básicos por parte de las mujeres campesinas

Por medio de metodologías participativas de las mujeres campesinas afiliadas a la Red de Mujeres Rurales, se logró analizar los procesos productivos y se determinaron las mejoras a introducir.

Posteriormente, se acompañó a los grupos productores de granos con el fin de fortalecer sus producciones, al igual que en la planificación de siembras y selección de variedades a utilizar entre las semillas adaptadas en la zona.

También se realizaron visitas periódicas a los lugares de siembra y se establecieron parcelas con semillas propias para incrementar la producción y fomentar el trabajo en equipo.

Se apoyó la puesta en funcionamiento de un centro de procesamiento de granos (CEPROMA), donado por el Instituto de Desarrollo Agrario (IDA) a uno de los grupos de mujeres.

Procesamiento de granos en la elaboración de galletas, mediante la transferencia de tecnología desarrollada en la Universidad de Costa Rica

Con este fin, se coordinó con las mujeres para realizar el proceso de transferencia de tecnología de la Universidad de Costa Rica para la producción de galletas de frijol y arroz.

Se estableció el proceso de capacitación para el procesamiento en la comunidad de Cariari y Pavones. Por último, se compró el equipo y se trasladó a las zonas. Las panaderías están funcionando en locales alquilados, pero ya se inició el proceso de construcción de las panaderías. 
Desarrollo de la capacidad de gestión y organización de las mujeres campesinas para la producción y comercialización del producto terminado

La metodología para el desarrollo de capacidades tuvo énfasis en la participación de los grupos locales de mujeres campesinas, mediante talleres para la construcción de los procedimientos, las normas de funcionamiento, la elaboración de instrumentos de registro y administración, la división de tareas en el flujo de trabajo y la toma de decisiones en la microempresa, a fin de propiciar un buen clima laboral y el desarrollo de las capacidades de las mujeres.

Se inició la elaboración de normas para el funcionamiento de las unidades productivas de granos y las panaderías.

\section{Desarrollo de una estrategia de mercadeo}

Se hizo un estudio de la introducción y aceptación del producto en los mercados potenciales. Se analizaron datos ya existentes sobre el tema. Posteriormente se formuló una estrategia de mercadeo con enfoque educativo-nutricional, usando como base la información recopilada mediante encuestas en el campo.

Se trabajó en el diseño de empaque y etiquetas, y se tomaron en cuenta los criterios de la Red de Mujeres Rurales a la hora de introducir en el mercado los productos terminados.

El proceso de campaña para la difusión y la comercialización de los productos procesados destacó elementos como la producción campesina, la participación de las mujeres y la nutrición.

\section{Resultados y discusión}

Fortalecimiento de la producción de granos básicos por parte de las mujeres campesinas

Se ubicaron productoras de maíz, arroz y frijol que habían conservado semillas de siembras anteriores, y se compró material para distribuir entre los grupos que sembrarían parcelas de granos básicos. Las mujeres se organizaron y establecieron tres parcelas comunitarias para la producción de arroz, frijoles y maíz.Al iniciar el segundo año del proyecto, se evaluó el desempeño del año anterior y se analizaron las condiciones de producción para el segundo año.

El IDA propuso la construcción de un Centro de Negocios para el Procesamiento y Mercadeo Agropecuario (CEPROMA) en el cantón de Los Chiles $y$, gracias a las mujeres organizadas en la Red de Mujeres Rurales (RMR), con el apoyo del proyecto, se gestionó para que se ubicara en Las Nubes de Los Chiles. El fin de este Centro es apoyar el manejo poscosecha de granos básicos en la zona. Posteriormente, se negoció, con éxito, que este fuera dado en concesión al grupo de mujeres.

Para la puesta en marcha del centro se solicitó capacitación al IDA, con el fin de que las mujeres conocieran el manejo de la maquinaria y el equipo recibidos. Además, se ha apoyado el proceso administrativo de la planta mediante la ejecución de talleres de capacitación. Asimismo, la planta presta el servicio de selección, limpieza, empaque y almacenamiento de granos a las comunidades vecinas de Coquital, Santa Elena, Medio Queso y Cristo Rey.

La organización de las mujeres para hacer siembras conjuntas en el terreno de alguna de ellas contribuyó a la toma de conciencia de que unidas pueden avanzar más.

El haber privilegiado el uso de semillas propias entre las productoras no solo permitió mantener el acervo genético de las especies, sino que hizo evidente la necesidad de conservar semillas criollas y la importancia de usarlas como material de siembra. Las experiencias en las zonas de trabajo con las semillas distribuidas por las instituciones del estado no han sido satisfactorias, ya que se han presentado problemas en su germinación y el desarrollo de las plantaciones, así como susceptibilidad a plagas y enfermedades, y problemas de bajo rendimiento de las cosechas.

Es por estas razones que algunas familias han optado por conservar semilla criolla para no depender de semillas de baja calidad, como las entregadas por funcionarios estatales. Al comprar semilla criolla para distribuir entre los grupos de mujeres, se le da valor al esfuerzo de conservar estos materiales y se reducen los riesgos de cosechas bajas. Las familias que conservan semillas criollas son pocas, pero al fomentar su siembra se estimula que un mayor número de personas se preocupe por guardarlas, lo 
que permite conservar la biodiversidad y disminuir la dependencia de insumos importados o de baja calidad.

Por otro lado, el fomento de la siembra de alimentos básicos que se logra en el proyecto, como son las hortalizas, el arroz, los frijoles y el maíz, aumenta la seguridad alimentaria de las familias de las mujeres participantes y permite mejorar su dieta. En la medida en que las familias no dependan de monocultivos de exportación para obtener sus ingresos, se aumenta su seguridad alimentaria. Cuando una mujer debe trabajar para una empresa agroexportadora para obtener sus ingresos, está expuesta a los vaivenes de los mercados internacionales para comer, mientras que cuando siembra los alimentos de su familia, está asegurando su comida.

Asimismo, las opciones de trabajo y el trato desigual a la mujer en el ambiente laboral, en general y del agro en particular, obligan a buscar opciones que les permitan obtener ingresos de forma justa. La opción de contar con una planta de procesamiento de los granos no solo genera ingresos a las mujeres sino que les permite vender los productos con un valor agregado que redunda en un mejor precio. Es una fuente de ingresos que permite, a la vez, diversificar el origen de estos.

\section{Procesamiento de granos en la elaboración de galletas}

Se apoyó el proceso de selección del terreno a comprar para la construcción de las panaderías en Campo Dos de Cariari y Pavón de Los Chiles. También se realizaron los trámites necesarios para la elaboración de planos, gestión de permisos de construcción y trámites diversos para iniciar la construcción de los locales de Cariari y Pavones.

Actualmente, los tres grupos, Cariari, Pavones y El Águila, están alquilando locales para las panaderías. En los dos primeros casos fue necesario un proceso de acondicionamiento para iniciar su funcionamiento, para lo que se contó con la asesoría de funcionarios de las universidades.

Se inició el proceso de transferencia de tecnología de la Universidad de Costa Rica, respaldado por un licenciamiento de la universidad sobre la marca "Pinticas". Las señoras de Cariari y Pavón fueron capacitadas en la elaboración de galletas.
El equipo de las panaderías de estos lugares fue trasladado a las zonas con sus respectivos seguros y con el respaldo de un convenio entre la Red de Mujeres Rurales y la Universidad Nacional. Se inició la capacitación a los grupos en el manejo del equipo.

Las panaderías se encuentran produciendo y comercializando los productos elaborados. La venta y distribución de sus productos se lleva a cabo en las comunidades donde están localizadas, no obstante, se ha iniciado la distribución en algunos establecimientos fuera de las comunidades.

También se hicieron los análisis pertinentes para la elaboración del etiquetado nutricional, y un estudio de almacenamiento utilizando el empaque que emplearán los grupos de mujeres para la comercialización de las galletas.

La industrialización de los granos les permite dar un paso en la cadena productiva, de modo que no se venda materia prima, sino producto elaborado. Esto obliga a las mujeres a capacitarse en mayor grado, no solo en los procesos industriales, sino también en los aspectos administrativos y organizativos. Es un reto que ha costado muchas horas de talleres, pero que ha valido la pena por el avance observado en la Red. Han surgido nuevos problemas, pero se han convertido en retos que han permitido el desarrollo de cada una de las participantes y de la Red en general.

Por otro lado, poder ofrecer a los niños, como meta primordial, un producto de alto valor nutritivo que sustituya la comida chatarra que suelen consumir es un logro que contribuye a la alimentación nutritiva, a la que tienen derecho las personas de esas comunidades y del país en general.

La transferencia de tecnología, desarrollada por el CITA de la Universidad de Costa Rica, es un excelente ejemplo de la generación de empresas a partir de desarrollos de las universidades. EI CITA cumple de esta manera su fin primordial de servir al país mediante el apoyo de grupos que han quedado marginados. La transferencia del conocimiento por medio de este tipo de alianzas es lo que da sentido al quehacer universitario en investigación.

La fortaleza que se logra con el desarrollo de empresas en manos de pequeños productores, especialmente si se trata de mujeres, es una 


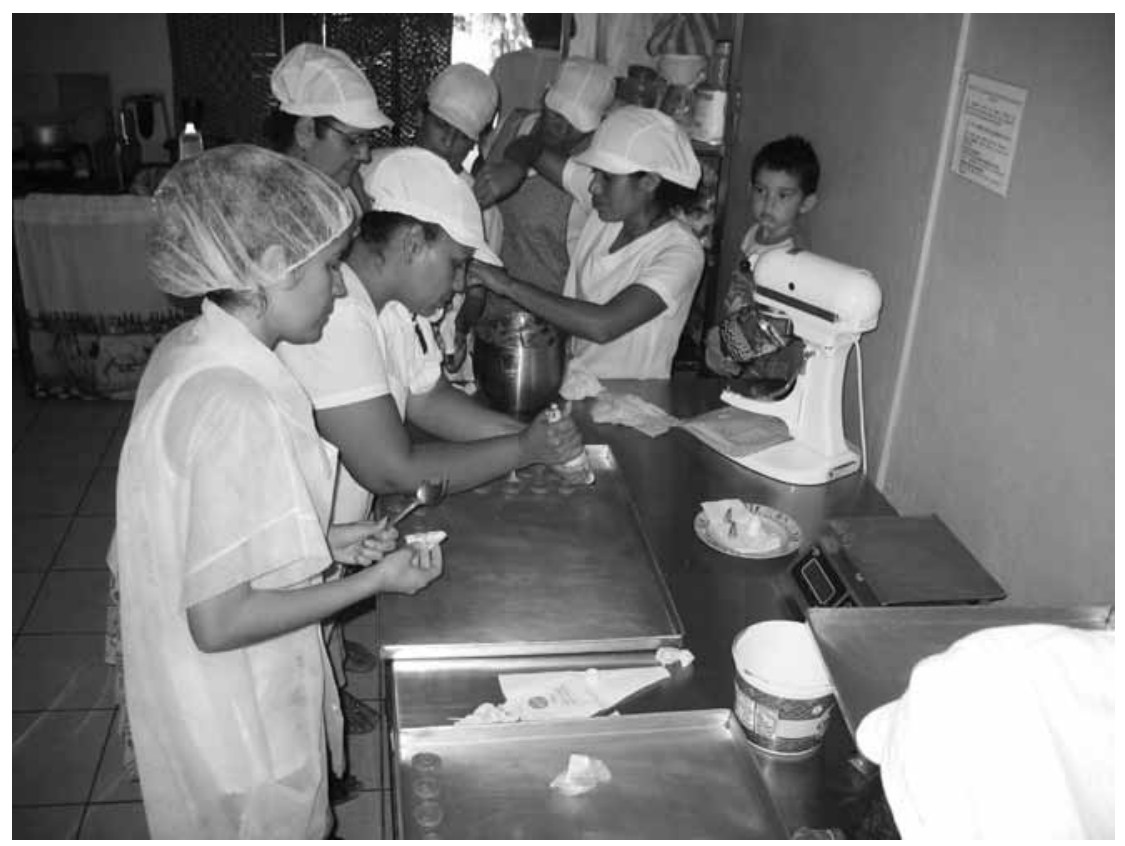

Figura 4. Capacitación para elaborar galletas.

contribución al crecimiento de la economía, pero, sobre todo, a lograr una distribución más justa de la riqueza. Costa Rica ha sido deficitaria en los índices de distribución de la riqueza, pero con este tipo de proyectos se contribuye a revertir esas deficiencias. Además, el hecho de hacer la transferencia a grupos ubicados en zonas rurales también contribuye a reducir la pobreza que se ha concentrado en esas regiones, especialmente en las que se incluyeron en este proyecto.

\section{Desarrollo de la capacidad de gestión y} organización de las mujeres campesinas para la producción y comercialización del producto terminado

Para continuar con el desarrollo de capacidades administrativas y organizativas de los grupos en la gestión de su microempresa, se realizaron visitas de planeamiento y evaluación de avance. También se organizaron y ejecutaron cursos y talleres de capacitación en temas administrativos, de comercialización, organización de la microempresa y producción agrícola. Asimismo, se analizó el proceso de producción, flujos, capacidad de producción y control de calidad. Posteriormente se evaluaron las actividades de capacitación y se incorporaron mejoras en el proceso.
Se elaboró el Manual de manejo de alimentos en panadería y se realizó una sesión de validación y revisión de este documento con las señoras. Se elaboraron tablas para el control de gastos, compras de materia prima, ventas y rendimiento diario de producción.

Con el propósito de iniciar el funcionamiento, se tramitó el permiso del Ministerio de Salud y se corrigieron las observaciones planteadas por este ministerio. También se hicieron las gestiones ante la CCSS para demostrar que se trata de una unidad productiva colectiva, donde no median relaciones patronales.

Por otra parte, se realizaron talleres de resolución de conflictos para el abordaje de diferencias en el interior de los equipos de producción.

Las señoras se incorporaron en la planificación y organización de la EXPO-UCR y en el Festival Mujeres Semillas y Arte.

La Red de Mujeres Rurales asumió el reto de liderar empresas relacionadas con la producción agropecuaria, como el procesamiento e industrialización de granos, además de continuar con la producción en sí misma. Esto ha requerido de un gran esfuerzo, mucho sacrificio de su tiempo familiar y personal, y un gran empeño por aprender cosas 


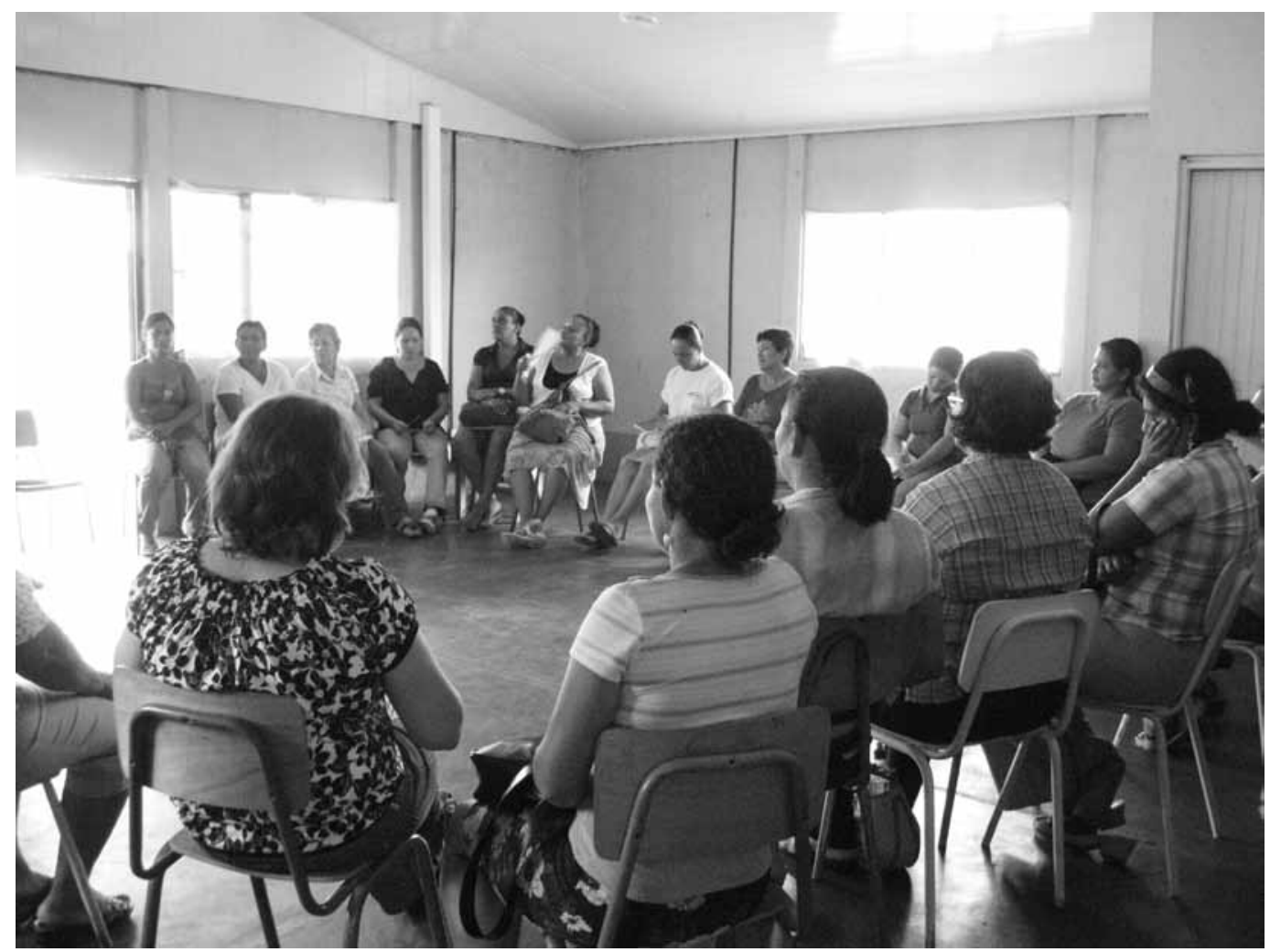

Figura 5. Taller de intercambio de experiencias entre grupos.

nuevas. El acompañamiento de las universidades en este proyecto ha facilitado el camino para lograr sus metas. El nivel de desarrollo de cada una de ellas y de la organización en general es una demostración de los avances logrados.

Por otro lado, un excelente ejemplo de los logros es la participación de las mujeres en foros, exposiciones y ferias, donde, con toda propiedad, han dado a conocer sus productos y su organización como un ejemplo para todas las mujeres de este país y de Latinoamérica.

En los talleres de evaluación de labores, ellas reconocen que les cuesta hablar en público y negociar con autoridades de instituciones $u$ organizaciones. Sin embargo, expresan la satisfacción de haber logrado superarse en alguna medida, unas más que otras, pero todas convencidas de que sí pueden hacerlo.

\section{Desarrollo de una estrategia de mercadeo}

Se hizo un estudio de campo sobre la aceptación de las galletas "Pinticas" en comunidades de las zonas de influencia de las panaderías de Cariari y Pavón, comunidades como Guápiles, Cariari, Semillero, La Rita, Siquirres Centro, Santa Rosa de Pocosol, Los Chiles Centro, Pavón y Ciudad Quesada, tanto en una muestra de abastecedores, como de comedores escolares y camiones repartidores. Además, se llevó a cabo un estudio sobre las preferencias de la población escolar y colegial, y los procedimientos de compra de los centros de educación.

Se participó en actividades como la Expo-UCR 20 I I, la Expo-Pococí 20 I I, Expo-Frijol y el Festival Mujeres, Semillas y Arte"; se abrieron espacios de demostración del producto y del proceso organizativo de la Red de Mujeres Rurales, que se complementaron con dinámicas y actividades que propiciaran la reflexión y facilitaran información sobre el perfil nutricional del producto. Los segmentos involucrados en estos espacios fueron, fundamentalmente, personas cercanas o vinculadas 


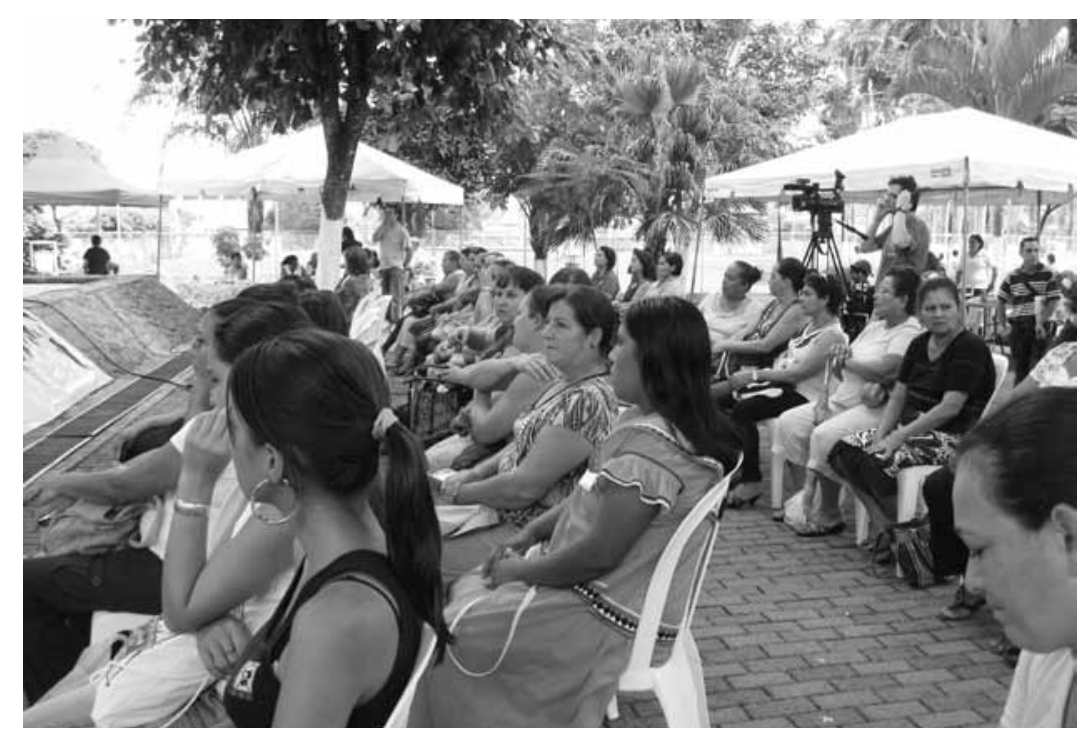

Figura 6. Festival Mujeres, Semillas y Arte, Pococí.

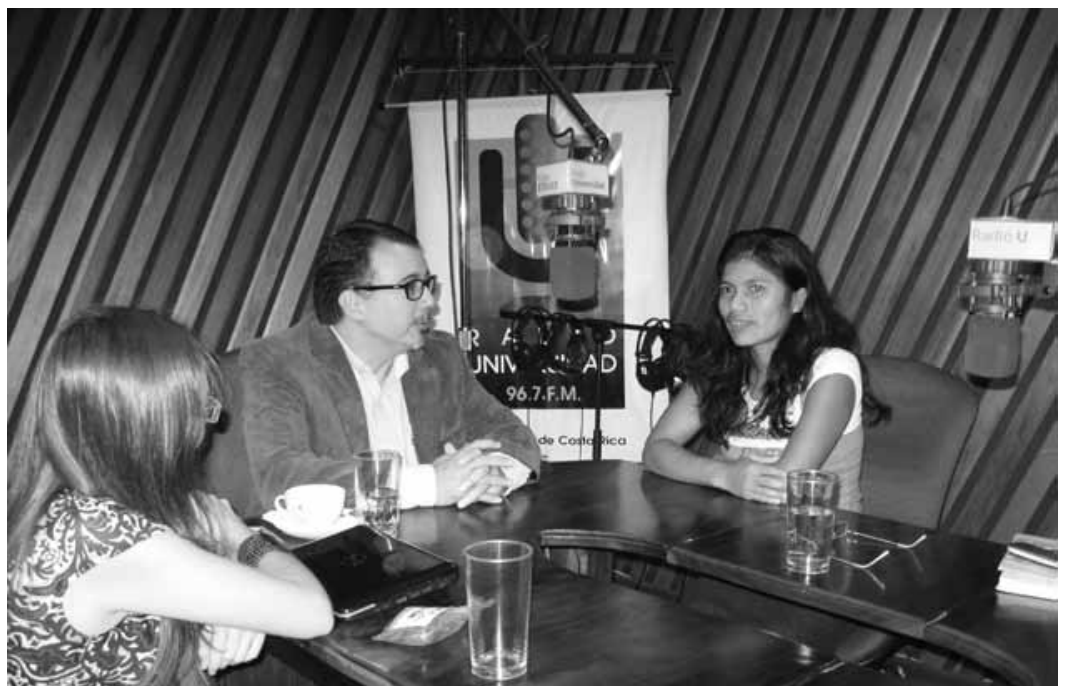

Figura 7. Participación en medios de Orfa Condega, presidenta de la Red.

a la dinámica de las universidades públicas, por un lado, y población local de la zona de Pococí.

El proceso de comercialización de los productos ha enfrentado a la Red a nuevos retos por superar. Aprender a negociar, a trabajar en equipo, a compartir las deudas y las ganancias, y a tolerar las diferencias son algunos de los caminos que han tenido que recorrer y que continúan recorriendo.

La estrategia de comercialización propuso un avance desde las comunidades propias hasta zonas más alejadas, que les ha enseñado la importancia de trabajar con calidad y en forma ordenada y sistemática. Este proceso está en sus inicios, pero es fundamental para el éxito de los proyectos que la Red se ha trazado.

\section{Conclusiones}

Se logró el avance esperado en el fortalecimiento de la producción de granos básicos, ya que mediante las visitas se ha fortalecido la producción de arroz y frijoles por parte de grupos de mujeres asociadas a 
la Red de Mujeres Rurales. A esto debe agregarse el fortalecimiento de la producción de maíz.

También es necesario fortalecer el control de calidad en las panaderías.

El proceso de extensión se ha venido desarrollando bajo una concepción de amplia participación en todas las áreas del proyecto de las mujeres pertenecientes a los grupos involucrados. Por ello, los talleres y las capacitaciones son el componente fundamental del proyecto. En los talleres se propicia una construcción colectiva de las acciones a emprender para que no resulte una intervención desde afuera. Esto ha permitido una apropiación del conocimiento, pero también una apropiación del proceso productivo y de las unidades productivas como tales.

Es fundamental la visión de proceso en la concepción metodológica, que trasciende el logro de los productos, ya que el proyecto mismo encara la exclusión de las mujeres del campo por su condición de campesinas y de mujeres. Cada participación, cada intervención de las mujeres y cada taller incluyen el desarrollo de las mujeres involucradas en la ubicación de las acciones que realizan en el entorno socio económico y sus derechos a ser agentes de desarrollo.

Se ha procurado que el componente de comunicación se desarrolle a manera de acompañamiento del proceso organizativo y productivo, sin perder de vista el cumplimiento de los objetivos concretos del proyecto, relacionados con el posicionamiento y venta del producto (las galletas). En este sentido, más que simplemente ocuparse por la promoción para la venta del producto, se ha tratado de apoyar las diferentes actividades organizativas y productivas, ya sea mediante la producción de mensajes (productos específicos de comunicación) como de la realización de actividades con un sentido educativo, y no solo con el interés de propiciar el mero consumo. Este trabajo se desarrolló de manera que se procurara estudiar la realidad en la que se está trabajando para realizar propuestas, tanto de actividades como de productos, coherentes con las necesidades y circunstancias de esas realidades.

\section{Bibliografía}

Bonilla,A. (2007). Patrimonio productivo de las mujeres campesinas. ¿Cambios o avances? Congreso Universitario de las Mujeres, Universidad de Costa Rica.

Bonilla, A.; Nielsen, S. (2007). Enhanced Bean Utilization in the US and Central America. Adding value to promote increased utilization and consumption. Bean/Cowpea collaborative Research Program. Regional partnerships to enhance bean/ cowpea consumption and production in Africa and Latin America. Final Report Purdue University and Universidad de Costa Rica. 2002-2007.

CONARE (20II). Estado de la Nación. XVII Informe Programa Estado de la Nación. Consejo Nacional de Rectores, San José, Costa Rica.

FAO-RUTA (20I0), Pequeños productores de granos básicos en América Central, estudio de Eduardo Baumeister. http://www. pesacentroamerica.org/biblioteca/ca_en_cifras.

Ministerio de Salud. (20II). Política Nacional para la Seguridad Alimentaria y Nutricional 2011-2021. San José: Ministerio de Salud. 54 pp.

Ministerio de Salud. (2010). Plan Nacional de Salud para 20102021. (p 54, 78). San José: Ministerio de Salud.

Naciones Unidas (1948). Declaración Universal de Derechos Humanos. Resolución 217 A (III) del 10 de diciembre de 1948 de la Asamblea General de las Naciones Unidas. http:// www.un.org/es/documents/udhrl

PRESANCA \& FAO. (20II). Centroamérica en cifras. Datos de seguridad alimentaria nutricional y agricultura familiar. Programa Regional de Seguridad Alimentaria para Centroamérica (PRESANCA II)-Organización de la Naciones Unidas para la Alimentación y la Agricultura (FAO)-Iniciativa América Latina y el Caribe Sin Hambre (IALCSH)-Programas Especiales para la Seguridad Alimentaria de Centroamérica (PESA). 27 pp.

Rebosio, Guillermo \& Melgar, Y. (200I). Seguridad Alimentaria, Género y Agroindustria. En Pequeñas Agroindustrial Rurales: Estrategias de Promoción y Consolidación Empresarial. Editorial CIED, Lima, Perú. I 16 pp.

Secretaría Ejecutiva de Planificación Sectorial Agropecuaria [SEPSA]. (2008). Plan Nacional de Alimentos Costa Rica: oportunidad para la agricultura nacional. San José, Costa Rica. SEPSA. 84 pp.

Soto, Y. (2008). Estimación de la vida útil de una galleta elaborada a base de frijol (Phaseolus vulgaris) por medio de un estudio de almacenamiento. Proyecto de graduación para optar por el grado de Licenciatura. Escuela de Tecnología de Alimentos. Universidad de Costa Rica. 